\title{
Neural Circuit Tuning Fly Visual Neurons to Motion of Small Objects II. Input Organization of Inhibitory Circuit Elements Revealed by Electrophysiological and Optical Recording Techniques
}

\author{
MARTIN EGELHAAF, ALEXANDER BORST, ANNE-KATHRIN WARZECHA, \\ SUSANNA FLECKS, AND ANKE WILDEMANN \\ Max-Planck-Institut für biologische Kybernetik, W-7400 Tübingen, Germany
}

\section{SUMMARY AND CONCLUSIONS}

1. The FD1-cell in the visual system of the fly is an identified visual interneuron that is specifically tuned to motion of small objects. In the companion paper it was shown that this response property is mediated by one of the two CH-cells, the VCH-cell, that inhibits the FD1-cell by GABAergic synapses. Iere the input organization of the two CH-cells is analyzed by both electrophysiological and optical recording techniques.

2. Both $\mathrm{CH}$-cells are excited by front-to-back motion in the ipsilateral and by back-to-front motion in the contralateral visual field. They respond maximally to binocular rotatory motion about the vertical axis of the animal. The latter response is only slightly less than the sum of the corresponding monocular response components. The relative contribution of the ipsi-and contralateral eye to the binocular response varies considerably between flies. In extreme cases it is dominated by either the ipsi- or the contralateral eyc. The two $\mathrm{CH}$-cells are not equally sensitive along the vertical axis of the eye. The DCH-cell has its sensitivity maximum in the dorsal part, the VCII-cell in the ventral part of the visual field.

3. The $\mathrm{CH}$-cells have two arborizations, a large one in the posterior part of the third visual neuropil, the lobula plate, and a smaller one in the ipsilateral ventrolateral brain. With the calcium-sensitive dye fura- 2 as an activity marker, it is analyzed which of these branches of the $\mathrm{CH}$-cells receive the ipsi- and contralateral motion input, respectively. During motion in the preferred direction within the ipsilateral visual field, calcium accumulates only in the $\mathrm{CH}$-cells' main arborization in the lobula plate but not in their branches in the ventrolateral brain, indicating that the arborization in the lobula plate is postsynaptic to the ipsilateral input. In contrast, contralateral motion in the preferred direction leads to calcium accumulation in both arborizations, suggesting that both are postsynaptic to contralateral input elements. During preferred direction motion in the upper or lower part of the ipsilateral visual field, calcium accumulates in only dorsal or ventral branches of the CH-cells' arborization in the lobula plate, respectively, revealing that their ipsilateral motion input is organized retinotopically. Because this arborization, most likely, is also the main output terminal of the CH-cells, it is both pre- and postsynaptic. This specific neuronal design is discussed with respect to its consequences for the mechanism of tuning the FDl-cell to motion of small objects.

INTRODUCTION

The main center of motion computation of the fly visual system is the posterior part of the third visual neuropil, the lobula plate. In addition to large numbers of small retino- topically arranged elements (Gilbert and Strausfeld 1992; Strausfeld 1976; Strausfeld and Gilbert 1992), there reside $\sim 50$ individually identifiable nerve cells with large dendritic trees, the so-called tangential cells (Hausen 1981; Hausen and Egelhaaf 1989). They are specifically tuned to various kinds of retinal motion patterns as are encountered by the animal when moving around in its environment. Part of them have been shown to play important roles in visual orientation behavior (Egelhaaf et al. 1988; Egelhaaf and Borst 1992; Hausen 1981; Hausen and Egelhaaf 1989; Hausen and Wehrhahn 1983). Most of these tangential cells acquire their characteristic motion selectivity by two basic processing steps. 1) With their extended dendritic trees, they spatially pool the output of large numbers of retinotopically organized local motion-sensitive elements. The major functional properties of these local elements could be inferred indirectly from the response characteristics of the tangential cells. (for review, see Borst and Egelhaaf 1989). By this retinotopic input the tangential cells become directionally selective to motion in large parts of the ipsilateral visual field. 2) The response properties of the tangential cells may be further shaped by excitatory or inhibitory input from other tangential cells of the ipsi- or contralateral lobula plate. These network interactions have been unraveled in detail for a particular cell type, the FD1cell that is most sensitive to the motion of small objects (Egelhaaf 1985a). As has been analyzed in the companion paper (Warzecha et al. 1993), the FD1-cell is inhibited via GABAergic synapses by the VCH-cell.

The VCH-cell is one of a pair of identified lobula plate cells, i.e., the CH-cells (Eckert and Dvorak 1983; Hausen 1976a,b). These cells cover with their main arborization the dorsal (DCH-cell) and ventral part ( $\mathrm{VCH}$-cell) of the lobula plate, respectively. Both $\mathrm{CH}$-cells have another smaller arborization in the ipsilateral ventrolateral brain. They respond best to binocular coherent large-field motion about the vertical body axis of the animal (Eckert and Dvorak 1983; Hausen 1976a) and hence under the conditions when the FD1-cell's response is reduced maximally (Egelhaaf 1985a).

On the basis of anatomic and electrophysiological evidences, the $\mathrm{CH}$-cells have been proposed to acquire their sensitivity to ipsilateral front-to-back motion not from retinotopically organized local input elements, but rather from the HS-cells. The HS-cells are another group of three identified tangential neurons, the HSN-, HSE-, and HSS- 
cell (Hausen 1982a,b), and thcy show similar reactions to ipsilateral motion as the $\mathrm{CH}$-cells (Hausen 1976a,b). The CH-cells' sensitivity to contralateral back-to-front motion was concluded to be mediated by tangential cells with dendritic trees in the contralateral lobula plate, the H1-(Eckert and Dvorak 1983; Hausen 1976a) and H2-cell (Hausen 1976a). Because, according to this scheme, these cells were believed to reconvey information on motion from the ventrolateral brain back to the lobula plate, they were termed centrifugal horizontal cells or $\mathrm{CH}$-cells.

The proposed ipsilateral input organization of the $\mathrm{CH}$ cells is in contrast to our experimental findings. In the companion paper it was shown by photoinactivating individual tangential cells of the lobula plate that the VCH-cell is the large-field inhibitor of the FD1-cell. Only ablation of the $\mathrm{VCH}$-cell led the FD1-cell to respond with a larger amplitude to large-field than to small-field motion. In contrast, inactivation of the HSE-cell did not affect the tuning of the FD1-cell to the motion of small objects (Warzecha et al. 1993 ). This finding can hardly be reconciled with the proposal that the HSE-cell represents a main ipsilateral input element of the VCH-cell.

These conflicting results made it necessary to reexamine the input organization of the $\mathrm{CH}$-cells. With electrophysiological and optical recording techniques, three particularly important aspects were analyzed: $l$ ) the selectivity of the $\mathrm{CH}$-cells to binocular large-ficld motion; 2) the $\mathrm{CH}$-cells' spatial sensitivity distribution along the vertical extent of the visual field; and 3 ) the postsynaptic sites of the $\mathrm{CH}$ cells' ipsi- and contralateral input.

\section{METHODS}

\section{Preparation and electrophysiological experiments}

The preparation of the flies, the intracellular recording techniques, and the evaluation of the electrophysiological data were as described in the companion paper (Warzecha et al. 1993). The only difference was that the tips of the electrodes were filled with $5 \%$ solutions of Lucifer yellow $\mathrm{CH}$ dissolved in $1 \mathrm{M} \mathrm{LiCl}$. The dye was injected iontophoretically ( -1 to $-3 \mathrm{nA} ; 10-20 \mathrm{~min})$. After the physiological experiments, this allowed to unambiguously identify the injected cell as either a $\mathrm{VCH}$ - or DCH-cell by their unique anatomic features (Eckert and Dvorak 1983; Hausen 1976a; Hengstenberg and Hengstenberg 1980). Because the arborizations of the lobula plate tangential cells are almost parallel to the surface of the brain, the main branches of the cell can be visualized under epifluorescence illumination in the living animal. All cells, the data of which were used in the present study, have been identified in this way. If an identification of a particular cell was not possible because its staining was too faint, the physiological data were discarded.

\section{Visual stimulation in electrophysiological experiments}

For visual stimulation in the electrophysiological experiments, two CRT screens (Tektronix 608) were used. The stimulus patterns were generated at a frame rate of $200 \mathrm{~Hz}$ by an image synthesizer (Picasso, Innisfree), which was controlled by programs written in ASYST (Keithley) on a PS /2-80 computer (IBM).

In the experiments aimed at analyzing the responses of the $\mathrm{CH}$ cells to monocular and binocular large-field motion, the monitors were placed symmetrically at an angle of $45^{\circ}$ with respect to the long axis and perpendicular to the horizontal plane of the animal. The fly was positioned in such a way that it faced the center of each screen with one of its eyes. The horizontal and vertical extent of the screens amounted to 48 and $35^{\circ}$, respectively. The stimulus pattern was a vertical sine-wave grating with a spatial wavelength of $12^{\circ}$ that was moved at a temporal frequency of $2 \mathrm{~Hz}$ in either horizontal direction. The mean luminance was $15.8 \mathrm{~cd} / \mathrm{m}^{2}$, the contrast $20 \%$.

In the experiments analyzing the vertical extent of the CH-cells' ipsilateral receptive fields, the two screens were placed on top of each other. Along their vertical axis, the screens were subdivided in two rectangular fields. The width and height of the four stimulus fields amounted to 38 and $26^{\circ}$, respectively. The horizontal location of the middle of the stimulus fields was in front of the right eye at $25^{\circ}$ with respect to the frontal midline of the animal. The vertical position of the fields was, from top to bottom, +20 , $+6,-19$, and $-33^{\circ}$, with $0^{\circ}$ representing the horizontal direction. Vertical sine-wave gratings (spatial wavelength, $9.5^{\circ}$; temporal frequency, $2 \mathrm{~Hz}$; mean luminance, $15.8 \mathrm{~cd} / \mathrm{m}^{2}$; contrast, 20\%) could be moved in either horizontal direction independently in the four stimulus fields.

\section{Optical recording}

To resolve the input regions of the $\mathrm{CH}$-cells, the fluorescent calcium indicator fura-2 (Grynkiewicz et al. 1985) was used. The methods are described in detail in a previous report (Borst and Egelhaaf 1992). T The electrode tips were filled by capillary action with a mixture of $2 \mathrm{mM}$ fura-2 frec acid (Molecular Probes) dissolved in $200 \mathrm{mM} \mathrm{KCl}$. The dye was injected iontophoretically into the cell $(-1$ to $-3 \mathrm{nA}, 10-30 \mathrm{~min})$. After the injection of the dye, some time (10-30 $\mathrm{min}$ ) was allowed for the dye to diffuse throughout the cell before the experiment started. The experiments were performed with an upright microscope (Zeiss Universal), a Zeiss UD20 long-distance objective (numerical aperture 0.56 ), and epifluorescence illumination (HBO $100 \mathrm{~W}$ mercury arc lamp ) with an appropriate filter combination for fura imaging [BP 380 or BP 349 excitation filters (bandwidth, $10 \mathrm{~nm}$ ), a FT 510 dichroic mirror, and a BP 500-530 barrier filter]. The imaging system consisted of a Peltier-cooled camera head (Photometrics CH 250A) with a CCD chip (Photometrics 512, ultra low dark current, $512 \times 512$ pixels), an clectronic unit (Photometrics CE $200 \mathrm{~A}$ equipped with a $50-\mathrm{kHz}$ 16-bit A/D converter) and a controller board (Photometrics NU 200). Images were acquired and evaluated by a software package (IPLab, Signal Analytics) on a Macintosh IIfx computer (Apple). The electrophysiological data obtained simultaneously with the optical data were acquired as described above. The data evaluation is described in RESULTS and the figure legends.

Under the microscope the fly faced the inside of a translucent hemispherical screen. The moving patterns were generated mechanically by a rotating striped cylinder with the light source (HBO $100 \mathrm{~W}$ mercury arc lamp) placed in its center. The patterns were projected onto the hemisphere from the outside and could be turned about the axis of projection by dove prisms. Two pattern windows with diameters of $\sim 20^{\circ}$, as seen by the fly, could be positioned on arbitrary sites on the hemisphere in the cell's receptive field. Within the windows, the patterns could be moved in any direction. The contrast frequency amounted to $1.8 \mathrm{~Hz}$.

\section{RESULTS}

\section{Electrophysiological responses to monocular and binocular motion}

Graded membrane-potential changes rather than spike activity represent the main response mode of the $\mathrm{CH}$-cells. They depolarize and hyperpolarize during motion in their preferred and null direction, respectively (Hausen 1976a). The responses of the two $\mathrm{CH}$-cells in the right optic lobe to 


\section{A}
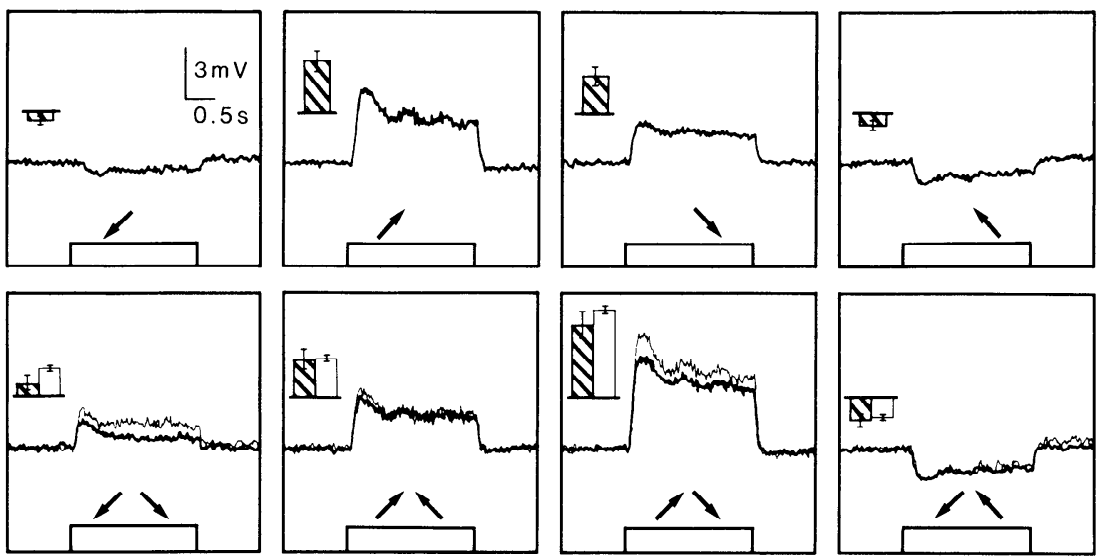

B
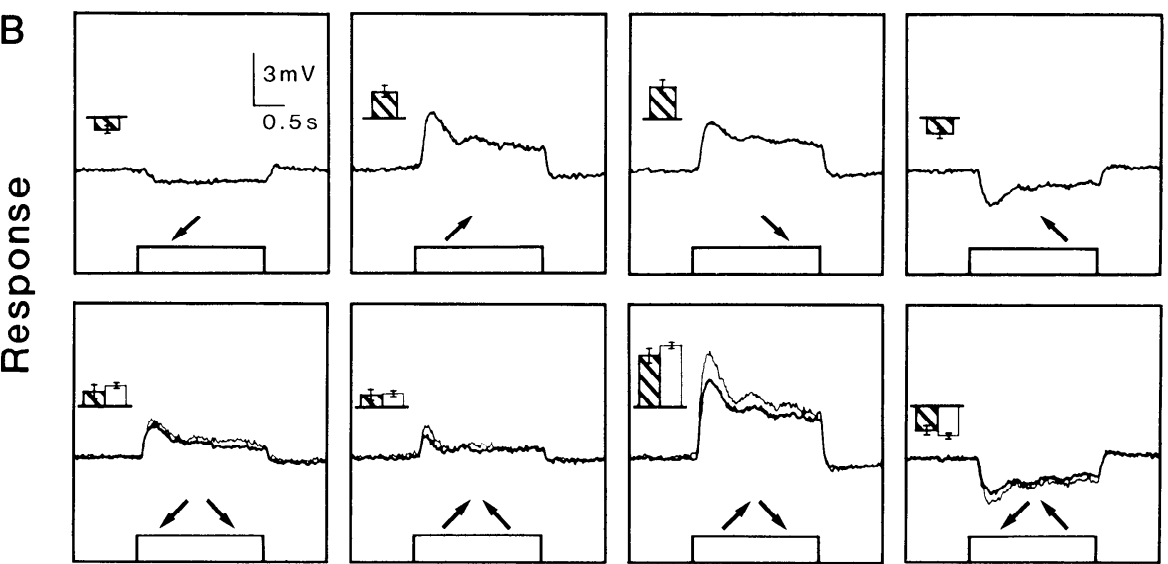

Time the various types of monocular and binocular horizontal large-field motion are shown in Fig. 1. As is characteristic of all motion-sensitive tangential cells in the third visual ganglion (Egclhaaf and Borst 1990), the CH-cells respond to the onset of motion with a transient response peak before the membrane potential settles at its steady-state level. In accordance with previous studies (Eckert and Dvorak 1983; Hausen 1976a), the cells are excited during both ipsilateral front-to-back motion and contralateral back-tofront motion and are inhibited during motion in the respective reverse directions. During binocular large-field motion the cells in the right lobula plate respond strongest when the pattern rotates clockwise about the vertical axis of the animal. The responses are much weaker during image cxpansion, i.e., both patterns move from the front to the back, or image contraction, i.e., both patterns move from the back to the front. When both patterns move counterclockwise, the $\mathrm{CH}$-cells in the right lobula plate are hyperpolarized. These responses are very similar to what is expected from a linear superposition of the corresponding response components induced by the monocular motion alone. This is true for the time-averaged amplitude of the steady-state response level as well as for the time course of the responses. It is mainly during clockwise motion in front of both eyes that the measured responses are slightly smaller than the ones predicted by linear combination of the monocular response components. This is expected from a synaptic saturation nonlinearity of the cell (for details about synaptic saturation in fly tangential cells, see Haag et al. 1992).
FIG. 1. Responses of the DCH- $(A)$ and $\mathrm{VCH}-$ cell $(B)$ in the right lobula plate to various types of monocular and binocular motion stimulation. Time course of the response is shown together with the mean steady-state response amplitude ( hatched bars) and SE. Cells were stimulated for 2 $s$ as is indicated by the bar below the time-dependent response traces. Stimulus conditions are symbolized by arrows that indicate the direction of monocular ( single arrow) or binocular motion (double arrow), respectively. Responses to binocular motion are shown together with the sum of the responses as calculated from the corresponding monocular response components (thin lines and open bars in the respective bottom rows). Responses are averages of responses from $6 \mathrm{DCH}$ cells and a total of 78 stimulus presentations and from $6 \mathrm{VCH}$-cells and a total of 95 stimulus presentations. Steady-state responses were obtained by averaging the time-dependent responses during the 2 nd half of motion stimulation. Both the DCH- and VCH-cells are excited most during clockwise binocular large-field motion and are inhibited most during binocular rotatory motion in the opposite direction.
It should be noted that the above conclusions are based on data averaged over several flies. By closer inspection of the individual response traces, a considerable amount of variability is found with respect to the relative contribution of the responses induced by ipsi- and contralateral motion alone to the overall response during binocular motion. This range of variability is illustrated in Fig. 2. Because no major differences are found between the $\mathrm{DCH}$ - and $\mathrm{VCH}$-cell, the data of both were pooled. The ratios between the responses to either contralateral back-to-front motion or ipsilateral front-to-back motion and the responses to rotatory binocular large-field motion were calculated. The relative frequency of occurrence of these ratios is plotted in Fig. 2. A ratio of 0 means that there is no response to the respective monocular motion; a ratio of 1 means that the response amplitudes induced by monocular and binocular motion stimulation are the same. Although sufficient data are not available to allow for an assessment of the distribution function of the response ratios, at least the extremes that may occur can be inferred from the data. There are flies where almost no depolarization of the $\mathrm{CH}$-cells is found during back-to-front motion in the contralateral visual field, and, consequently, the entire activation of the cell during binocular clockwise motion is due to the ipsilateral motion input. The other extreme is cells that do not exhibit much activation during ipsilateral front-to-back motion; hence their activity induced by binocular clockwise motion is largely a consequence of their contralateral motion input alone. 


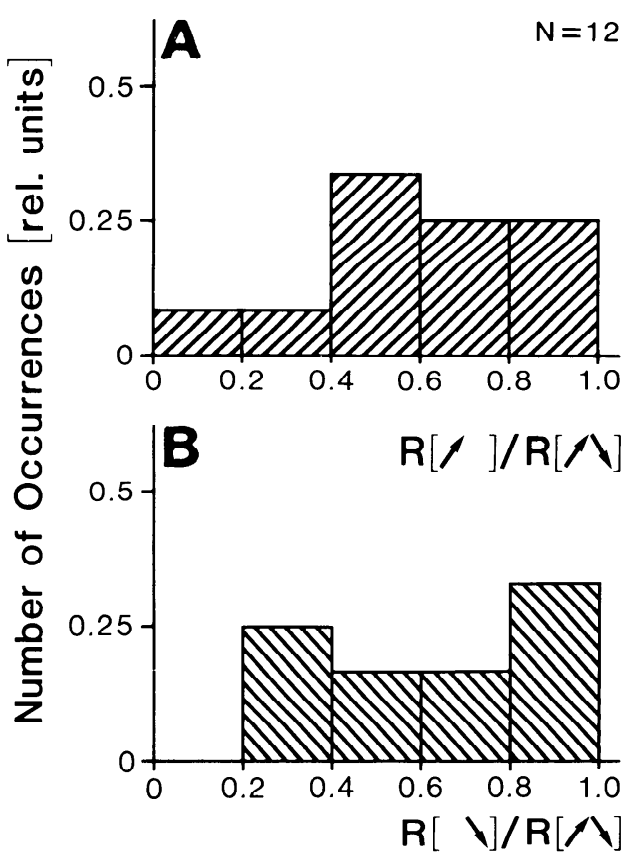

FIG. 2. Variability of the $\mathrm{CH}$-cell responses. Because no major differences are found in the responses of the $\mathrm{DCH}$ - and $\mathrm{VCH}$-cell under the stimulus conditions described in the legend of Fig. 1, the data obtained from both cell types were pooled (same data as displayed in averaged form in Fig. 1). Diagrams show the relative frequency of occurrence of the ratio between the mean steady-state responses of the different flies to either contralateral back-to-front motion $(A)$ or ipsilateral front-to-back motion $(B)$ and the responses to rotatory binocular large-field motion. A ratio of 0 indicates that there is no response to the respective monocular motion; a ratio of 1 means that the response amplitudes induced by monocular and binocular motion stimulation are the same. Data reveal a wide range of variability in the relative contribution of the responses induced by ipsi-and contralateral motion to the overall response induced by binocular motion.

This variability should be reflected in the strength of inhibition of the FD1-cell under the various conditions of largefield motion. Therefore it would be desirable to compare the variability found in the $\mathrm{CH}$-cell responses with the variability in the responses of the FDl-cell. In principle, this comparison should allow us to infer the transmission characteristic of the inhibitory synapse between the $\mathrm{VCH}$ - and FDl-cell. However, this is not possible, because most available data are from extracellular recordings of the FD1-cells (Egelhaaf 1985a; Warzecha et al. 1993). Hence the FD1cells could not be identified anatomically, and only functional criteria were used to assess whether a particular cell was an FD1-cell. This identification procedure may lead to an unintended reduction in variability. For instance, a cell was only regarded as an FD1-cell, if there was significant inhibition by back-to-front motion in the contralateral visual field (Warzecha et al. 1993). However, given the variability of the $\mathrm{CH}$-cell responses, some FDl-cells are expected to occur that do not show this inhibition during contralateral stimulation. Indeed, occasionally, cells were encountered that otherwise had functional properties of an FD1-cell (location of receptive field, smaller response amplitude during binocular large-field motion than during small-field motion ). However, no significant inhibition induced by contralateral motion could be detected. Accordingly, these cells were not classified as FD1-cells and were not included in the further analysis.
If the proposal of Hausen (1976a,b) were correct that the $\mathrm{CH}$-cells do not receive their ipsilateral motion input from retinotopically organized elements (see INTRODUCTION), the CH-cells' receptive fields cannot be deduced from their anatomic branching pattern in the lobula plate. Therefore we determined in four vertically displaced areas the spatial sensitivity distribution of the two $\mathrm{CH}$-cells' ipsilateral receptive fields (Fig. 3). The vertical extent, in particular, of the VCH-cell's receptive field is important for an understanding of the mechanism tuning the FD1-cell to smallfield motion, because the FD1-cell has a pronounced sensitivity maximum in the ventral part of the visual field.

Both $\mathrm{CH}$-cells are not equally sensitive within their rcceptive field to ipsilateral motion at different vertical positions but have a clear sensitivity maximum. On both sides of the maximum, the sensitivity falls off steeply. What is most important in the present context is that the response maxima are displaced along the vertical axis of the eye. Whereas the DCH-cell is most sensitive above the equator of the eyc, the VCH-cell has its sensitivity maximum below the equator. Hence both $\mathrm{CH}$-cells are not equivalent but mediate information about large-field motion in different parts of the ipsilateral visual field.

\section{Optical monitoring of the synaptic input sites of the CH-cells}

The $\mathrm{CH}$-cells have two arborizations, one in the lobula plate and the other in the ventrolateral brain (Eckert and Dvorak 1983; Hausen 1976b). In principle, both arborizations could be input sites either of the ipsi- or contralateral input elements or of both of them. An investigation of the input sites of the $\mathrm{CH}$-cells was particularly important, because the photoinactivation study on the potential large-

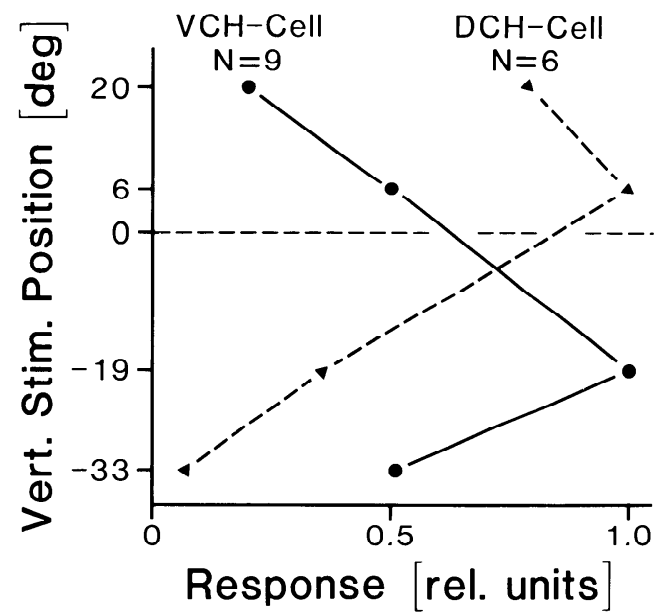

FIG. 3. Spatial sensitivity distribution of the DCH- $(\mathbf{\Delta - - - \Lambda})$ and VCH-cell (-C) along the vertical extent of the ipsilateral eye. The steady-state response amplitude was obtained as described in the legend of Fig. 1 and was measured in 4 vertically displaced nonoverlapping areas in the $2 \mathrm{CH}$-cells' ipsilateral receptive fields. Note the orientation of the axes. Mean positions of the stimulus patterns are given on the $y$-axis. 7ero degrees ( see dashed horizontal line) indicates the equatorial plane of the fly's visual field. Data are averages obtained from $6 \mathrm{DCH}$-cells and a total of 60 stimulus presentations and from $9 \mathrm{VCH}$-cells and a total of 92 stimulus presentations. The responses were normalized to their maximum. The $\mathrm{SF}$ calculated from the raw data are after normalization $<0.1$ for all stimulus conditions. Whereas the DCH-cell is most sensitive above the equator of the eye, the VCH-cell has a clear sensitivity maximum below the equator. 

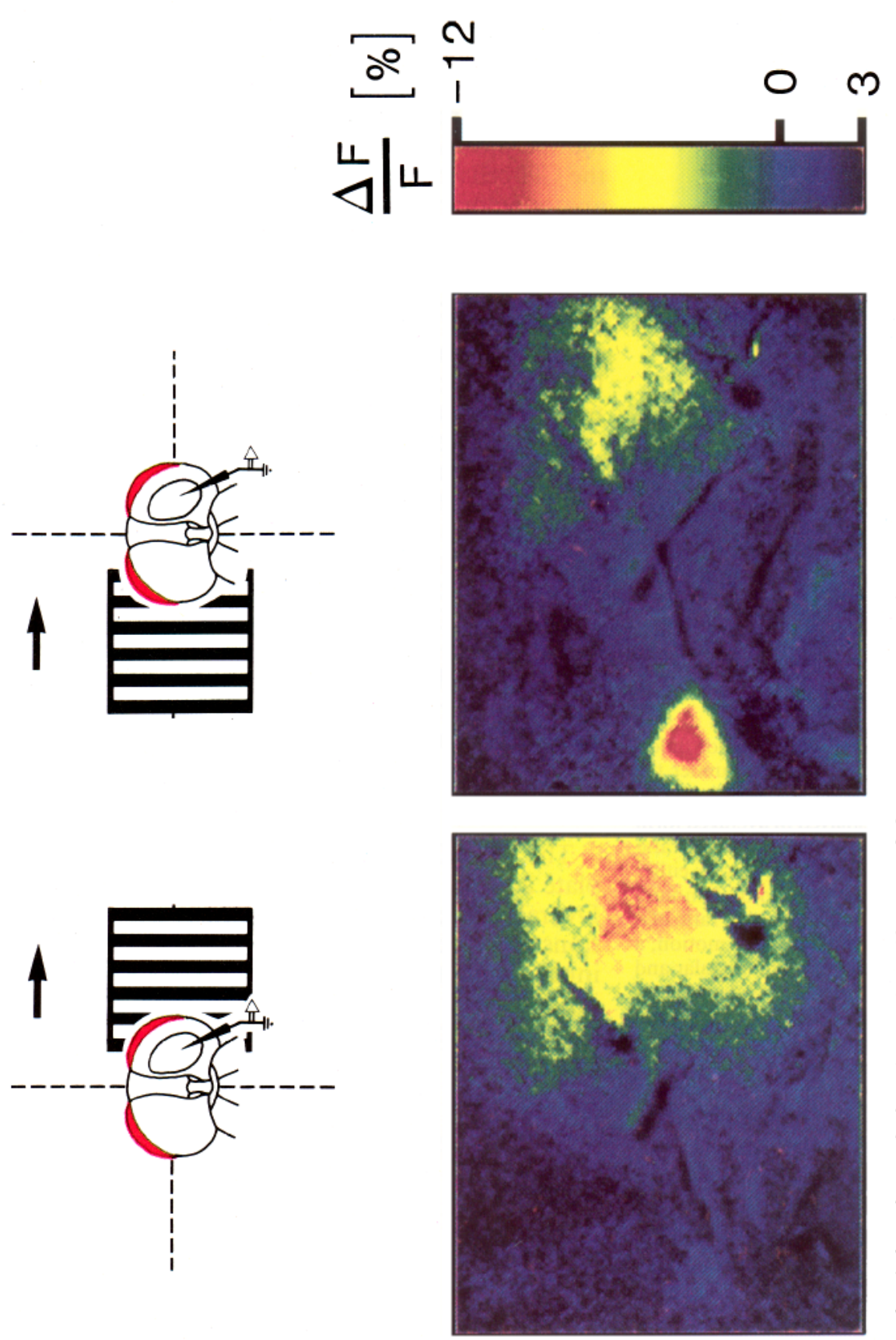

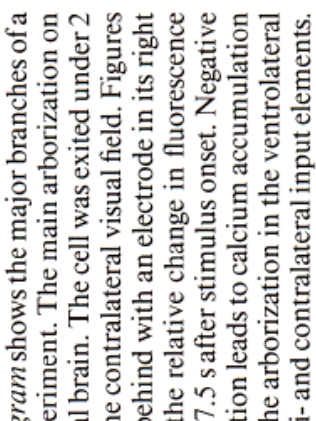

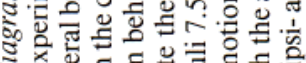

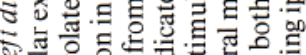

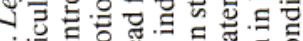

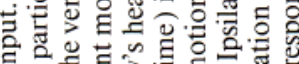

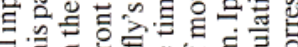

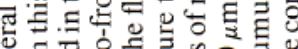

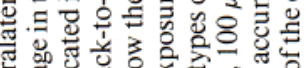

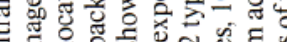

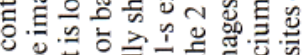

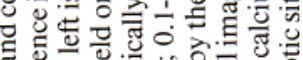

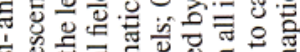

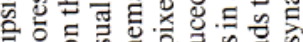

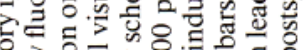

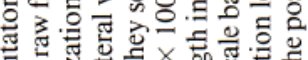

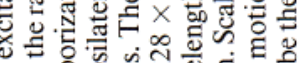

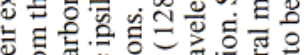

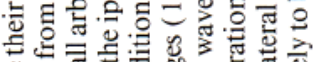

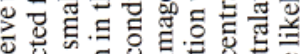

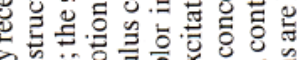

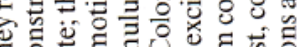

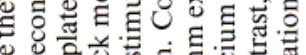

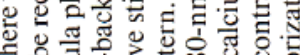

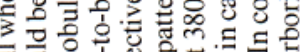

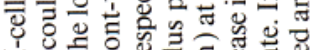

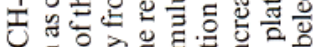

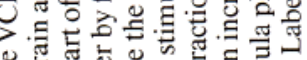

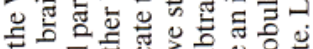

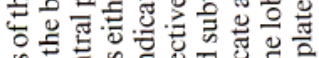

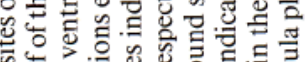

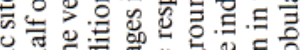

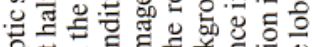

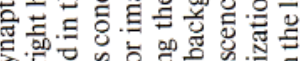

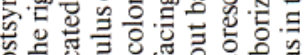

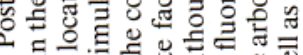
. +

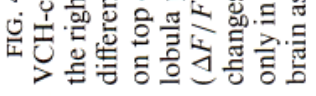

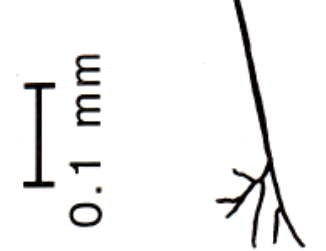



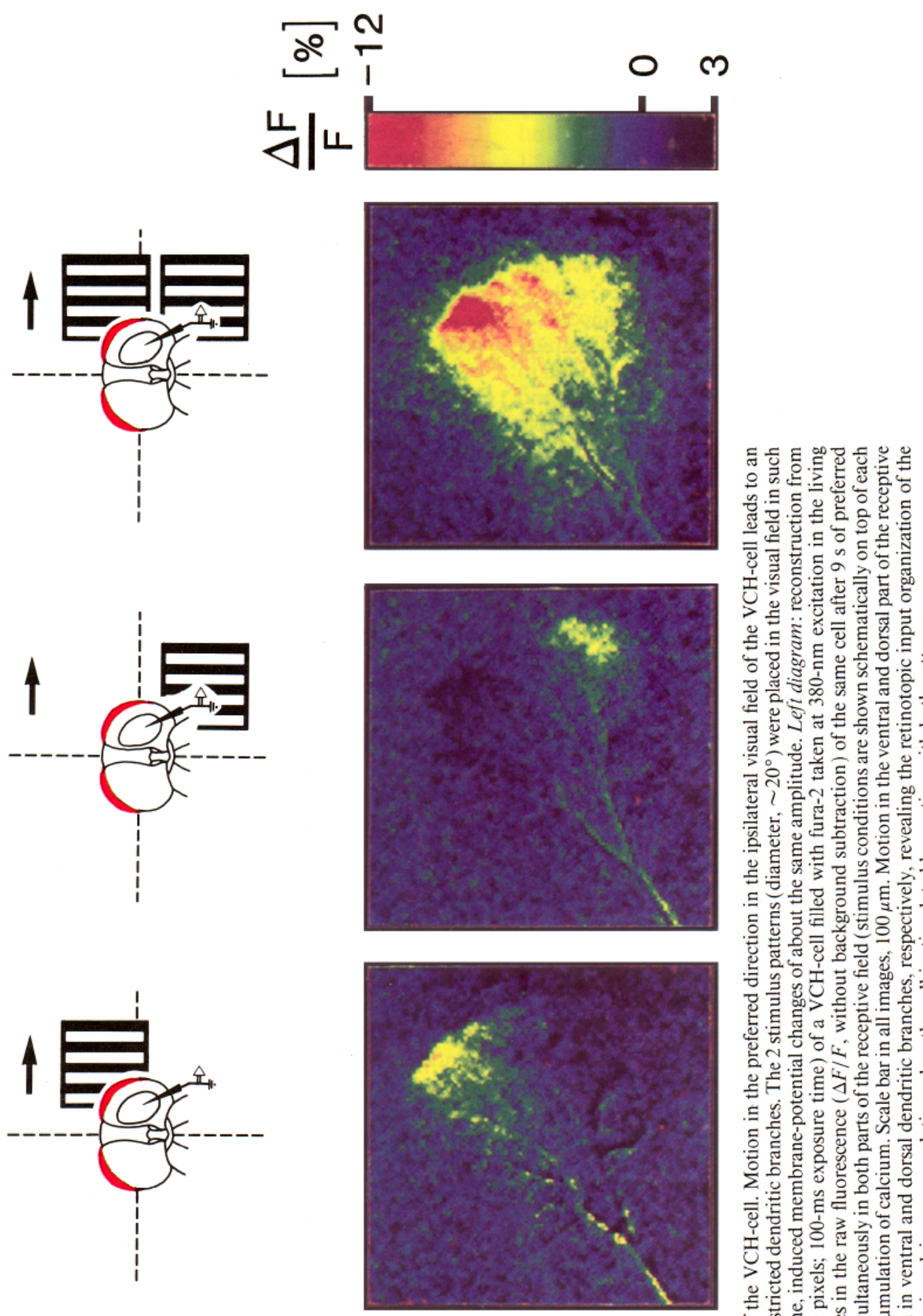

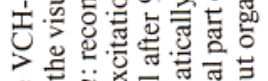

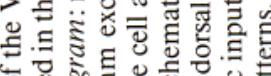

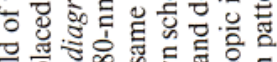

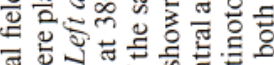

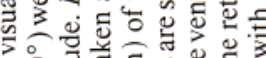

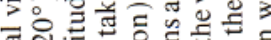

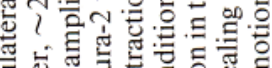

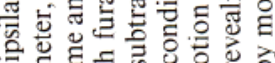

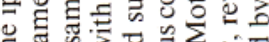

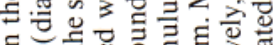

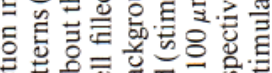

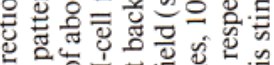

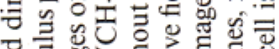
远寻管

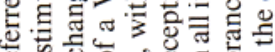

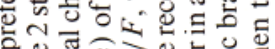

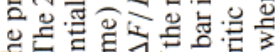

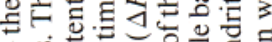

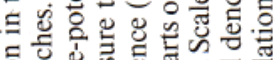

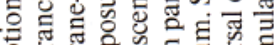

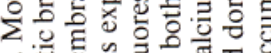

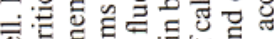
षृ ह

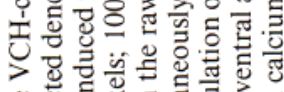
记

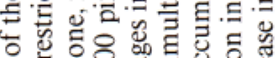

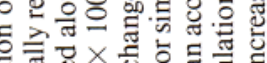

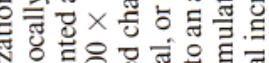

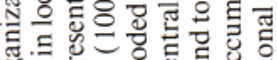

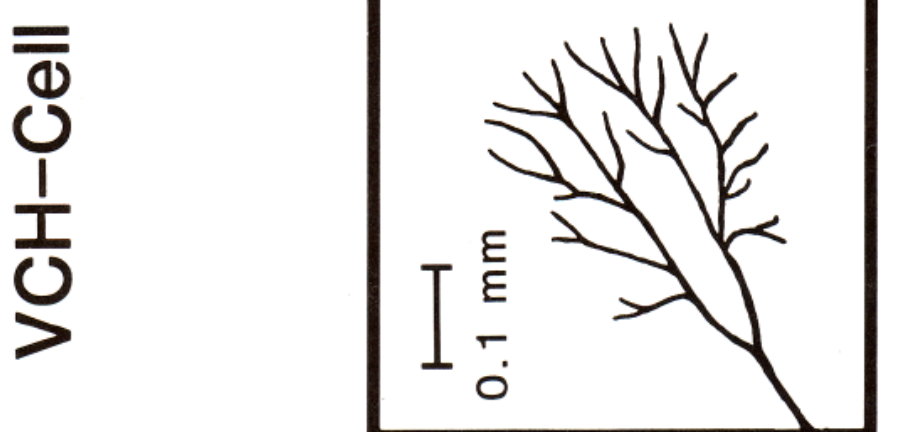

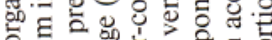

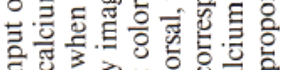
을 हี่

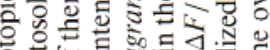

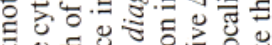

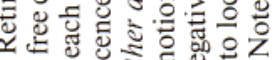

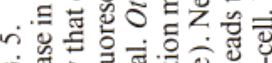

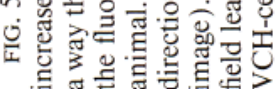


ficld inhibitor of the FD1-cell (Warzecha et al. 1993) casted doubt on the input circuitry of the $\mathrm{CH}$-cells as proposed by Hausen (see INTRODUCTION). Therefore we wanted to find out through which arborization the $\mathrm{CH}$-cells receive their ipsilateral input.

The only method available, at present, to map the synaptically induced spatial activity distribution within single cells is the optical recording technique. Recently, we applied this technique in the fly visual system and were able to monitor the spatiotemporal changes in calcium concentration as induced by motion stimulation (Borst and Egelhaaf 1992). The optical recording technique can be employed in the semi-intact fly where the cells can still be activated and inhibited by their natural synaptic input. This is possible because the arborizations of the tangential cells in the lobula plate are more or less two dimensional, lying $<50 \mu \mathrm{m}$ below and almost parallel to the surface of the brain. We could show that, in addition to the presynaptic terminal and the cell body, only selected parts of the dendrite of various types of tangential cells are activated by motion stimuli restricted to small parts of the visual field, visualizing, for the first time, the retinotopic input organization of these interneurons (Borst and Egelhaal 1992).

In the present study we applied this technique to the $\mathrm{CH}$ cells. In each experiment a single cell was filled with the fluorescent calcium indicator fura-2. Visually induced intracellular calcium accumulation served here exclusively as a marker of the sites that are activated by synaptic input. In addition, the dye allowed us to identify the injected cell not only by physiological criteria but also owing to its characteristic anatomic structure. A sequence of pictures of the cell was taken at intervals of $1.5 \mathrm{~s}$. Five control pictures were taken preceding stimulus presentation followed by 20 pictures during stimulus motion. Another 15 pictures were taken after cessation of pattern motion. From these raw fluorescence pictures the relative fluorescence changes induced by motion stimulation were calculated. Thereby the first picture was used as a reference. A decrease in relative fluorescence corresponds to an increase in cytosolic calcium and thus to an activation of the cell. The pictures shown in Figs. 4 and 5 represent in a color-coded form the relative fluorescence changes of the cell induced by motion stimulation, with blue indicating the resting condition and red the largest fluorescence changes (for details, see Borst and Egelhaaf 1992).

In the experiment illustrated in Fig. 4, a VCH-cell was injected with fura- 2 and subsequently activated either by front-to-back motion in the ipsilateral visual field or by back-to-front motion presented to the contralateral eye (see schematic diagrams above color plots). Ipsilateral motion leads to calcium accumulation only in the main arborization in the lobula plate and not in the arborization in the ventrolateral brain. In contrast, contralateral motion induces an increase in calcium concentration simultaneously in both arborizations. It should be noted that, in the labeled part of the cell, no distinct dendritic branches can be seen. The labeling rather appears to be more or less homogeneous. The main reason for this is that, in vivo, only the main dendritic branches can be visualized in the raw fluorescence images. The large number of fine distal branches of the $\mathrm{CH}$-cells' arborizations usually could not be recognized as is suggested by comparison with anatomic data with the use of conventional dyes and sectioned material (Hausen 1976a; Hengstenberg and Hengstenberg 1980). Hence, from the labeling of the whole area of the dendritic tree, it is suggested that calcium accumulates mainly in the fine branches. This is also the reason why a quantitative estimate of the calcium concentration cannot be given here, because this is only possible in those branches of the cell that can be clearly distinguished against their background (for a detailed discussion of this point, see Borst and Egelhaaf 1992). This, however, does not affect the qualitative conclusions drawn here. The experiment was done in three VCH-cells and two DCH-cells with similar results in each case. Although there is some variability in the relative strength of labeling of the two arborizations of the $\mathrm{CH}$-cells during contralateral motion, two points become clear on the basis of these data. 1) Because the small arborization in the ventrolateral brain is only labeled during contralateral motion, although the cell is depolarized under both stimulus conditions, it is suggested that it represents a postsynaptic site receiving its input exclusively from contralateral input. This is in contrast to the input organization of the $\mathrm{CH}$-cells as proposed previously (see INTRODUCTION). 2) The labeling of the main arborization in the lobula plate during motion in front of either eye may indicate that it is postsynaptic to both ipsi- and contralateral input elements.

These conclusions are further substantiated in experiments where the cell injected with fura- 2 was stimulated by horizontal motion of a grating pattern in either the dorsal or ventral part of its ipsilateral receptive field, respectively, or in both regions simultaneously (see schematic diagrams above the color plots in Fig. 5). This experiment was done with four VCH-cells and two DCH-cells with a similar outcome in each case. In the example shown in Fig. 5, a VCHcell was filled with dye. When the cell is activated by motion stimulation in the dorsal part of the receptive field, only the dorsal dendrites are labeled. Accordingly, only the ventral dendritic branches are labeled when the motion stimulus is presented in the ventral part of the visual field. When motion is presented in both stimulus fields, the entire dendritic tree appears labeled. It should be noted that the labeling induced by motion in both stimulus areas is much larger than the summated activity patterns as induccd during motion in each area alone. This feature was observed in most $\mathrm{CH}$-cells investigated so far and will be considered in the DISCUSSION. In conclusion, the activity labeling induced by motion in different parts of the visual field clearly speaks in favor of the interpretation that the $\mathrm{CH}$-cells receive their ipsilateral motion input from retinotopically organized input elements rather than from other large-field elements. Without direct activation by retinotopic input, it is hard to imagine how the fluorescence changes could be restricted to those parts of the arborization that cover the corresponding retinotopic area of the lobula plate.

\section{DISCUSSION}

\section{Specificity of the CH-cells to binocular rotatory large-field motion}

As a pair, the $\mathrm{CH}$-cells jointly cover with their dendritic trees the dorsal and ventral part of the lobula plate (Hausen 
1976a). They are activated by front-to-back motion in the ipsilateral visual field and by back-to-front motion in the contralateral visual field but respond strongest to rotatory binocular motion in front of both eyes. In accordance with earlier studies (Eckert and Dvorak 1983; Hausen 1976a), the response amplitude during rotatory binocular largefield motion in the preferred direction of the $\mathrm{CH}$-cells was found to be only slightly less than the linear sum of the corresponding response components induced by monocular motion alone (Fig. 1).

The specificity of the $\mathrm{CH}$-cells to rotatory binocular large-field motion can be further inferred by comparing the response to this stimulus condition with the response amplitudes induced during stimulation with either monocular motion or combinations of different directions of motion in front of the two eyes. Interestingly, positive responses are also induced during both binocular image expansion and contraction. However, in these cases the response amplitudes are much smaller than during binocular rotatory large-field motion (Fig. 1). Hence the $\mathrm{CH}$-cells are tuned quite specifically to binocular rotatory image motion as occurs when the animal turns about its vertical body axis. Cells with a similar specificity to binocular large-field motion have been found in bees (Ibbotson 1991). This hints at similar synaptic interactions that tune these cells to binocular rotatory motion. Of course, a higher degree of specificity to this retinal motion pattern could have been achieved by more sophisticated interactions between the motion signals originating from both eyes. For instance, in the cervical connective of flies, cells have been found that respond exclusively to binocular motion stimuli, either expanding or rotatory ones (Borst 1991; A. Borst and U. Thiemann, unpublished observations ).

Interestingly, the $\mathrm{CH}$-cells are much more specific to rotatory binocular large-field motion than the so-called HScells that reside also in the lobula plate of the fly (Hausen 1982a,b). Although the HS-cells are excited, as the CHcells, during front-to-back and back-to-front motion in the ipsi- and contralateral visual field, respectively, we found in accordance with previous studies (Hausen 1976a, 1982a,b) that the HS-cells do not respond with larger response amplitudes to binocular rotatory large-field motion than to frontto-back motion in the ipsilateral visual field alone (Egelhaaf and Borst 1992). This is interesting because the HS-cells, as output cells of the optic lobes, are believed to mediate compensatory optomotor turning reactions induced by deviations of the fly from its flight course (Egelhaaf et al. 1988; Hausen 1981; Hausen and Egelhaaf 1989; Hausen and Wehrhahn 1983, 1990). Behavioral studies on tethered flies showed that, in the optomotor response, motion input from both eyes summates in a more or less linear way, thus leading to response amplitudes of about twice the size in case of binocular rotation as compared with monocular front-to-back motion alone (Borst et al. 1991). Hence it has to be assumed that the optomotor pathway assumes its specificity to rotatory binocular large-field motion by some later processing stage, e.g., by computing the difference of the responses of the HS-cells from both sides of the brain. In the case of the $\mathrm{CH}$-cells, such interactions have to take place within the optic lobes, because they are intrinsic elements of the visual system.

\section{Synaptic input organization of the $\mathrm{CH}$-cells}

In previous optical recording experiments with the calcium-sensitive dye fura- 2 , we found motion-induced activity in the dendritic tree of HS- and VS-cells of the lobula plate (Borst and Egelhaaf 1992). This allowed us to functionally locate postsynaptic areas of these cells. In the present study optical recording has been employed to demonstrate that motion in the visual surround is also topographically projected onto the dendritic tree of the $\mathrm{CH}$-cells, indicating that the CH-cells, like the HS- and VS-cells, receive their ipsilateral motion input from retinotopically organized local elements. In this way, the $\mathrm{CH}$-cells become sensitive to motion in the ipsilateral visual field, just as has been concluded for all other tangential cells in the lobula plate analyzed so far. These data are in conflict with earlier conclusions based on anatomic and physiological considerations (Hausen 1976a,b, 1981). Originally, it was tentatively presumed that the $\mathrm{CH}$-cells receive their ipsilateral input from the HS-cells rather than from retinotopically organized input elements (see INTRODUCTION). The main reason for this was the finding that ipsilateral stimulation of the $\mathrm{CH}$-cells leads to graded membrane potential changes without any distinct synaptic potentials. Because the HScells show similar graded potential changes and their axon terminals are in close contact with the processes of the $\mathrm{CH}$ cells in the ventrolateral brain, it seemed possible that both cell types are synaptically connected (Hausen 1976a,b). In a later study, this proposal was further specified, and the two $\mathrm{CH}$-cells were claimed not to receive input from all three HS-cells in the same way. Spatial sensitivity measurements indicated that the I)CH-cell receives input from both the HSN- and HSE-cell and that the VCH-cell receives input from the HSS- and HSE-cell (Hausen 1981). These conclusions got further support from the finding that the arborization of the $\mathrm{CH}$-cells in the lobula plate shows presynaptic specializations ( see bclow). Ncither of these points provides sufficient evidence for the hypothesis that the $\mathrm{CH}$ cells receive input from the HS-cells. 1) Graded membranepotential changes without distinct excitatory postsynaptic potentials (EPSPs) are also expected if the $\mathrm{CH}$-cells receive input from a large number of retinotopically organized cells, spiking or nonspiking ones. This is because the activity of these input elements are likely not to be synchronized. Because neighboring input elements receive input from different spatial phases of the moving stimulus pattern, their activity should be phase shifted (Egelhaaf ct al. 1989). Therefore discrete EPSPs should be smoothed out to a large extent. Accordingly, smooth graded potential changes during ipsilateral motion are also found in other tangential cells, such as the HS-cells, that were concluded to receive their input from local retinotopic elements. 2) The receptive fields of the $\mathrm{VCH}$-cell and the DCH-cell are expected to be roughly similar to the combined receptive fields of part of the HS-cells even if they are not connected to them but receive their input from retinotopic elements. Because the main arborizations of the VCH- and DCH-cell cover the ventral and dorsal part of the lobula plate (Eckert and Dvorak 1983; Hausen 1976a), it is expected in the case of either input organization that they are most sensitive to motion in the lower and upper part of the visual field, re- 
spectively. If we take these qualifications and our optical recording data into account, it appears to be safe to conclude that the ipsilateral input of the $\mathrm{CH}$-cells is organized in a retinotopic way.

The optical recording experiments furthermore suggest that the $\mathrm{CH}$-cells receive their contralateral input through two distinct regions, namely their processes in the lobula plate and the arborizations in the ventrolateral brain. This input is likely to be mediated by tangential cells from the contralateral optic lobe. Indeed, there is some electrophysiological evidence from double recordings that two identified neurons, the H1-cell (Eckert and Dvorak 1983; Hausen 1976a) and H2-cell (Hausen 1976a), that are both sensitive to back-to-front motion in the contralateral visual field, synapse onto $\mathrm{CH}$-cells; however, published data are either from the DCH-cell or a $\mathrm{CH}$-cell that was not filled with dye to allow for an anatomic identification (Eckert and Dvorak 1983; Hausen 1976a). On the other hand, the responses of both $\mathrm{CH}$-cells to contralateral motion can hardly be distinguished, indicating that they receive their contralateral input from elements with very similar functional characteristics (see Fig. 2) (see also Hausen 1976a). Hence the assumption that the VCH-cell receives excitatory input from the $\mathrm{H} 1$ - and $\mathrm{H} 2$-cell is, at present, the most plausible possibility and therefore is schematically illustrated in the wiring diagram of Fig. 6. However, the caveat remains that this connection scheme should not yet, without further electrophysiological data, be taken for granted. Despite this qualification, the wiring scheme shown in Fig. 6 is supported by our optical recording experiments where those arborizations of the $\mathrm{CH}$-cells are labeled during contralateral motion that overlap with the output regions of the $\mathrm{H} 1-$ and $\mathrm{H} 2$-cell. Because the axon terminal of the H2-cell overlaps with the arborizations of the $\mathrm{CH}$-cells in the ventrolateral brain, their synaptic connections are likely to be located there. That these arborizations of the $\mathrm{CH}$-cells are postsynaptic rather than presynaptic is in accordance with their anatomic fine structure, which shows typical postsynaptic specializations, i.e., spinelike profiles emerging sporadically from the main dendritic branches (Hausen 1976a). When optically monitoring the stimulus induced fluorescence changes during contralateral motion stimulation, these arborizations of the $\mathrm{CH}$-cells were strongly labeled (Fig. 4).

The only overlap between the axon terminals of the H1-cell and arborizations of the $\mathrm{CH}$-cells are within the lobula plate (Eckert and Dvorak 1983; Hausen 1976a). Interestingly, during contralateral motion stimulation also, these arborizations were labeled, although the labeling was usually weaker than of the dendritic branches in the ventrolateral brain. However, the optical recording data alone could not assign the lobula plate arborization of the $\mathrm{CH}$-cells as an input region for contralateral input signals, because this part of the neuron is likely to represent also the output region of the $\mathrm{CH}$-cells ( see below), and calcium accumulation there could just be presynaptic calcium needed for transmitter release. In addition to this excitatory input, the $\mathrm{CH}$-cells have been concluded to be inhibited by another contralateral element that is sensitive to front-to-back motion (not shown in Fig. 6). This has been inferred from the small inhibitory postsynaptic potentials that can be ob-
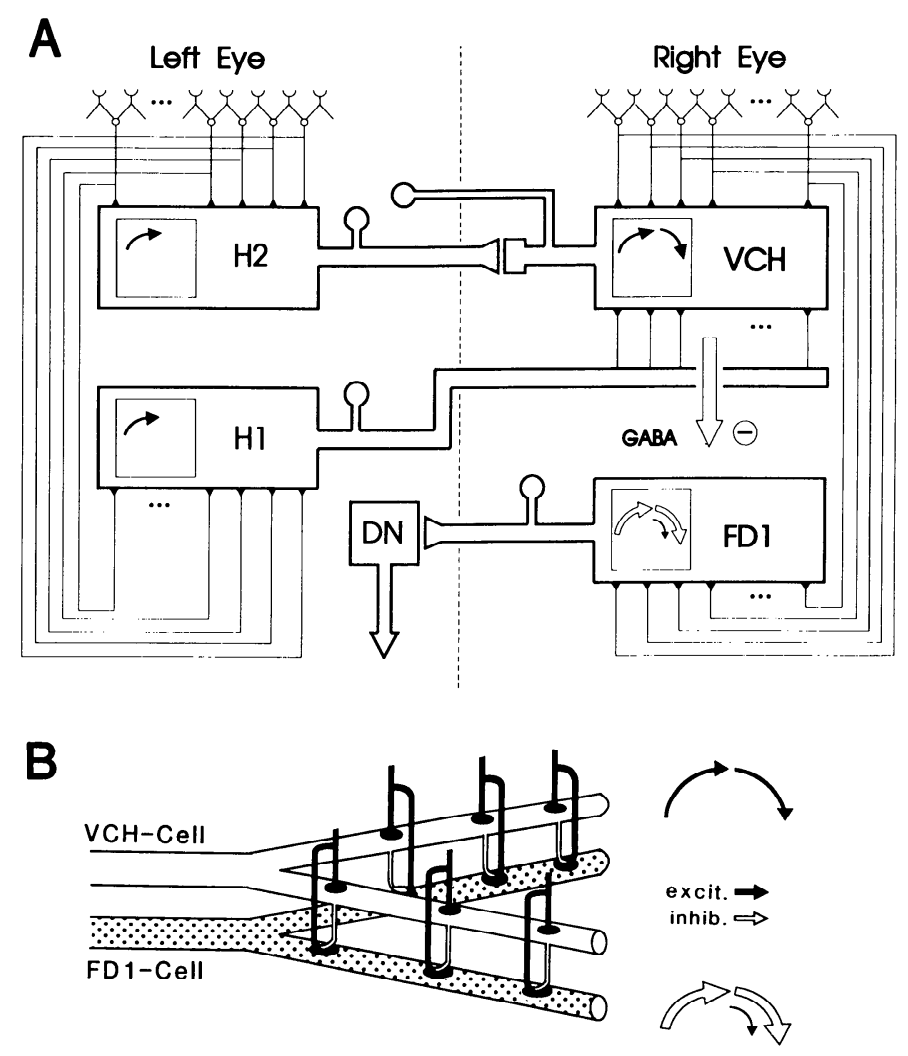

FIG. 6. Working hypothesis of the ncural circuit responsible for tuning the FDl-cell to small moving objects. $A$ : the $\mathrm{VCH}$-cell is assumed to receive its ipsilateral input from retinotopically organized local motion-sensitive elements and its contralateral input from tangential cells of the other optic lobe. Whereas the $\mathrm{H} 1$-cell is assumed to synapse on the extended lobula plate arborization of the $\mathrm{VCH}$-cell, the $\mathrm{H} 2$-cell is assumed to synapse on the arborization in the ventrolateral brain (for a discussion of the evidence for these connections, see text). The VCH-cell inhibits the FD1cell that, in turn, is assumed to mediate via descending neurons (DN) the detection and fixation of small moving objects. The inhibitory connection between the $\mathrm{VCH}$ - and FD1-cell is indicated by an arrow. Arrows in the boxes within the schematic dendrites of the various cells indicate the stimulus conditions that lead to either maximum excitation (filled arrows) or, in the case of the FD1-cell, to maximum inhibition (open arrows); large arrows indicate large-field motion; the small arrow in the schematic FDIcell indicates small-field motion. Arrows in the left and right halves of the boxes denote motion in the left and the right visual field, respectively. Dashed vertical line indicates the midline of the brain. Thin branches of the axons with their circular endings represent the cell body fibers and the cell bodies, respectively. $B$ : schematic diagram of the details of the inhibitory interaction between the VCH-cell and the FD1-cell. Shown are 2 dendritic branches of the VCH-cell and the FDl-cell, respectively. Both cells receive input from retinotopically organized local motion-sensitive elements. Although there are both excitatory and inhibitory retinotopic input elements, only the excitatory ones are shown. Hypothetically, the VCH-cell is shown to inhibit with its dendritic branches the FDI-cell indirectly via its retinotopic input elements. Filled and open synaptic connections represent excitatory and inhibitory synapses, respectively.

served in the response traces during contralateral motion from the front to the back (Hausen 1976a). The inhibitory input element of the $\mathrm{CH}$-cells has not yet been identified.

\section{Synaptic output of the CH-cells}

If the arborizations of the $\mathrm{CH}$-cells in both the ventrolateral brain and the lobula plate are postsynaptic, where are 
the output terminals of the cells? There are two lines of evidence that the main arborizations in the lobula plate are not only post- but also presynaptic. 1) They show characteristic varicose swellings ("blebs") (Hausen 1976a; Hengstenberg and Hengstenberg 1980) that are commonly interpreted as manifestations of presynaptic specializations (Altman and Tyrer 1980; Hausen et al. 1980; Muller and McMahan 1976; Watson and Burrows 1982). 2) It has been claimed that the $\mathrm{CH}$-cells inhibit the retinotopic input elements of the ipsilateral $\mathrm{H} 1$-cell thereby increasing its direction selectivity. Because the $\mathrm{H} 1$-cell only arborizes in the lobula plate, this inhibition is probably accomplished in the lobula plate (Hausen 1981). Hence it appears to us most likely that the VCH-cell reduces the activity of the FD1-cell also with its dendritic arborization in the lobula plate. In this case, retinotopic input channels activate the various dendritic branches of the FD1-cell and simultaneously inhibit these branches via the VCH-cell. This implies that the inhibitory interactions between the $\mathrm{VCH}$ - and the FD1-cell are localized. Whether these interactions are direct dendrodendritic interactions or indirect via the retinotopic input elements of the FD1-cell, as is schematically illustrated in Fig. 6, is not yet clear, because the relevant anatomic data that may allow this distinction are still missing.

Given this and the fact that the lobula plate arborization of the VCH-cell exceeds the area of the FD1-cell dendrite considerably in its horizontal extent, it is well conceivable that the FD1-cell is not the only FD-cell that is inhibited by the VCH-cell. In fact, other FD-cells, such as the FD4-cell, whose dendrites spread more toward the proximal part of the lobula plate and whose spatial sensitivity peaks accordingly in more lateral parts of the visual field (Egelhaaf 1985a), might be inhibited by the VCH-cell and, perhaps, also the DCH-cell. This, however, is pure speculation because no physiological and anatomic data exist so far supporting such connections.

Combined pre- and postsynaptic arborizations of neurons are not unusual in nervous systems. Dendrodendritic interactions have been reported, for instance, for various cell classes ( mitral cells, granule cells, periglomerular cells) in the vertebrate olfactory bulb (Shepherd 1977) and for amacrine cells in the vertebrate retina (Famiglietti 1983; Kolb and Nclson 1981 ). Although possible functional consequences of the specific synaptic design are being discussed, for instance, of the amacrine cells with respect to motion detection (Borg-Graham and Grzywacz 1992; Vaney et al. 1989), none of these ideas has been verified experimentally, so far.

\section{Possible computational properties of the circuit}

The cellular scheme for small-ficld tuning of the FD1-cell presented here (see Fig. 6) resembles in many respects the original model proposed to underly figure-ground discrimination behavior of the fly (Poggio et al. 1981; Reichardt et al. 1983 ). Most notably, the inhibitory action of a hypothetical "pool cell" sensitive to binocular large-field motion onto the element sensitive to small-field motion was concluded not to be direct onto the small-field element itself, but presynaptically on its retinotopic input elements. The advantage of this sort of wiring over an axoaxonal inhibition may lie in the fact that in the latter case the retinotopic input signals onto the FD1-cell would be spatially pooled before being inhibited by the spatially pooled signal through the $\mathrm{VCH}$-cell. In these spatially pooled signals, however, increases in pattern size are completely confounded with increases, for instance, of pattern contrast or velocity. In contrast, with the inhibition acting on the presynaptic elements of the FD1-cell, information about pattern size still is retained in the activity pattern of the input. We currently investigate by computer simulations of compartmental models what consequences the different wiring schemes have for the small-field tuning of the FD1-cell. These simulations indicate that, in the case of an axoaxonal interaction, the optimum object size of the FD1-cell is expected to be shifted toward larger sizes when pattern contrast or velocity goes down. In contrast, the problem of confounding pattern size with other stimulus parameters is alleviated, at least to some extent when the large-field inhibitor interacts with the input channels of the FD1-cell in a distributed way.

Besides the features that the original model for figureground discrimination has in common with the cellular circuit proposed for the FDl-cell, there are several differences. As already pointed out in a previous study (Egelhaaf 1985b), the large-field inhibitor or pool cell proposed to explain the behavioral responses was assumed to be insensitive to the direction of motion, whereas the inhibitory element of the FD1-cell that now turned out to be the VCHcell is directionally selective. The postulation of a directionally unselective pool cell in the original model comes from the behavioral observation that, in the early experiments studying figure-ground discrimination by relative motion in the fly, the figure was not discriminated if it moved in counterphase to the background pattern (Reichardt and Poggio 1979; Reichardt et al. 1983). This finding matches well the response properties of the FD4-cell that has been concluded to be inhibited by a directionally unselective large-field inhibitor or two inhibitors with opposite polarity (Egelhaaf 1985a,b). However, in a later behavioral analysis studying figure-ground discrimination for a range of oscillation frequencics (Rcichardt ct al. 1989), the behavioral responses during antiphase motion of figure and ground differed from the earlier studies. Under the stimulus conditions employed in this study, the fly could, on average, discriminate the figure. Although the reasons for the discrepancy between the two behavioral studies are not yet clear (for a detailed discussion, see Reichardt et al. 1989), the new results hint at a mechanism mediating figure-ground discrimination that relies on a large-field inhibitor that is directionally selective, just as was found for the FD1-cell.

In all cellular models proposed so far to underly figureground discrimination behavior of the fly (Reichardt and Poggio 1979; Reichardt et al. 1983, 1989) or the specific response properties of the various FD-cells (Egelhaaf $1985 \mathrm{~b}$ ) the inhibitory signal is first spatially pooled before it exerts its inhibitory influence on the retinotopic input channels of the FD-cell. Our present hypothesis is different 
in this respect. This is because the branches of the $\mathrm{VCH}$-cell in the lobula plate are assumed to be both post- and presynaptic. Such a scheme is only equivalent with the former models if the lobula plate arborization of the VCH-cell is isopotential. Given the very thin branches of this arborization (Hausen 1976a; Hengstenberg and Hengstenberg 1980 ), this assumption is rather unlikely, although no physiological measurements concerning the biophysical parameters of the cell are available so far. Hence it appears to us more plausible that the inhibitory signals mediated by the VCH-cell are, at least to some extent, localized. This hypothesis is corroborated by the localized calcium accumulation that is induced during ipsilateral motion stimulation in restricted parts of the visual field and the finding that this calcium entry is through voltage-dependent calcium channels in the dendrite (Egelhaaf and Borst 1992).

In this context, one observation from our optical recording experiments might turn out to be important. The calcium accumulation has been found to increase overproportionally with an increasing size of the stimulus pattern (Fig. 5 ). If we assume that the motion-induced calcium is also involved in mediating transmitter release, small-field motion would lead to only weak inhibition of the FI)l-cell. In contrast, large-field motion should then lead to an overproportional increase in transmitter release and hence to a much stronger inhibition of the FD1-cell. If these considerations were correct, the nonlinear increase in calcium accumulation with increasing size of the stimulus pattern would reflect a nonlinear relationship between pattern size and the strength of inhibition. Interestingly, theoretical considerations have revealed that this sort of nonlinearity greatly improves the efficiency of a mechanism tuning cellular elements to small-field and relative motion (Borst and Egelhaaf 1993). Whether this conclusion turns out to be correct is currently being analyzed by further electrophysiological and optical recording experiments.

We are grateful to C. Gilbert, K. G. Götz, and W. Reichardt for critically reading and discussing the manuscript.

Address reprint requests to $\mathrm{M}$. Egelhaaf.

Received 6 April 1992; accepted in final form 30 September 1992.

\section{REFERENCES}

ALTMAN, J. S. AND TYRER, N. M. Input synapses on a locust sensory neurone revealed by cobalt-electron microscopy. Brain Res. 189: 245250,1980

Borg-Graham, L. J. and Grzywacz, N. M. A Model of the Direction Selectivity Circuit in Retina: Transformations by Neurons Singly and in Concert. New York: Academic. In press.

Borst, A. Fly visual interneurons responsive to image expansion. Zool. Jahrh. Physiol. 95: 305-313, 1991.

Borst, A., BuchstäBer, $\Gamma$., AND EgelhaAF, M. Spatial integration of visual motion information in the optomotor response of the housefly. In: Synapse-Transmission-Modulation, edited by N. Elsner and $\mathrm{H}$. Penzlin. Stuttgart: Georg Thieme Verlag, 1991, p. 262.

Borst, A. AND EgelhaAf, M. Principles of visual motion detection. Trends Neurosci. 12: 297-306, 1989.

Borst, A. AND EgelhaAF, M. Direction selectivity of fly motion-sensitive neurons is computed in a two-stage process. Proc. Natl. Acad. Sci. USA 87: 9363-9367, 1990.

BORST, A. AND EGELHAAF, M. In vivo imaging of calcium accumulation in fly interneurons as elicited by visual motion stimulation. Proc. Natl. Acad. Sci. USA 89: 4139-4143, 1992.

Borst, A. AND EgElhaAF, M. Processing of synaptic signals in fly visual interneurons selectively responsive to small moving objects. In: Brain Theory-Spatio-Temporal Aspects of Brain Function. Amsterdam: Elsevier. In press.

ECKERT, H. AND DVORAK, D. R. The centrifugal horizontal cells in the lobula plate of the blowfly, Phaenicia sericata. J. Insect Physiol. 29: $547-560,1983$

EgELHAAF, M. On the neuronal basis of figure-ground discrimination by relative motion in the visual system of the fly. II. Figure-detection cells, a new class of visual interneurones. Biol. Cybern. 52: 195-209, 1985a.

EgELHAAF, M. On the neuronal basis of figure-ground discrimination by relative motion in the visual system of the fly. III. Possible input circuitries and behavioural significance of the FD-cells. Biol. Cybern. 52: 267$280,1985 b$.

EGELHAAF, M. How do fly FD-cells acquire their sensitivity to small-field motion. Naturwissenschaften 77: 182-185, 1990.

EgElHAAF, M. AND BORST, A. Transient and steady-state response properties of movement detectors. J. Opt. Soc. Am. A 6: 116-127, 1989. Errata J. Opt. Soc. Am. A 7: 172, 1990.

EGElHAAF, M. AND Borst, A. Motion computation and visual orientation in flies. Comp. Biochem. Physiol. In press

EgelhaAf, M., Borst, A., AND PILz, B. The role of GABA in detecting visual motion. Brain Res. 509: 156-160, 1990.

EGElHAAF, M., BORST, A., AND REICHARDT, W. Computational structure of a biological motion detection system as revealed by local detector analysis in the fly's nervous system. J. Opt. Soc. Am. A 6: 1070-1087, 1989.

EgelhaAf, M., Hausen, K., Reichardt, W., and Wehrhahn, C. Visual course control in flics relics on neuronal computation of object and background motion. Trends Neurosci. 11: 351-358, 1988.

FAmiglietTi, E. V. On and off pathways through amacrine cells in mammalian retina: the synaptic connections of "starburst" amacrine cells. Vision Res. 23: 1265-1279. 1983.

Gilbert, C. AND Strausfeld, N. J. Small-field neurons associated with oculomotor and optomotor control in muscoid flies: functional organization. J. Comp. Neurol. 316: 72-86, 1992

Grynkiewicz, G., Poenie, M., And Tsien, R. Y. A new generation of $\mathrm{Ca}^{2+}$ indicators with greatly improved fluorescence properties. J. Biol. Chemists 260: 3440-3450, 1985.

HaAg, J., EgelhaA,, M., and Borsi, A. Dendritic integration of visual motion information in the fly. Neurosci. Lett. 140: 173-176, 1992.

Hausen, K. Struktur, Funktion und Konnektivität bewegungsempfindlicher Interneurone im dritten optischen Neuropil der Schmeissfliege Calliphora erythrocephala ( $\mathrm{PhD}$ dissertation). Tübingen, Germany: Univ. of Tübingen, 1976a.

HAUSEN. K. Functional characterization and anatomical identification of motion sensitive neurons in the lobula plate of the blowfly Calliphora erythrocephala. Z. Naturforsch. 31c: 629-633, 1976b.

HAUSEN, K. Monocular and binocular computation of motion in the lobula plate of the fly. Verh. Dtsch. Zool. Ges. 74: 49-70, 1981.

HAUSEN, K. Motion sensitive interneurons in the optomotor system of the fly. I. The horizontal cells: structure and signals. Biol. Cybern. 45: 143156, 1982a.

HaUSF, K. Motion sensitive interneurons in the optomotor system of the fly. II. The horizontal cells: receptive ficld organization and response characteristics. Biol. Cybern. 46: 67-79, 1982b.

HAUSEN, K. AND EGELHAAF, M. Neural mechanisms of visual course control in insects. In: Facets of Vision, edited by D. Stavenga and R. Hardie. Berlin: Springer-Verlag, 1989, p. 391-424.

HaUSEN, K. AND Weilriairin, C. Microsurgical lesion of horizontal cells changes optomotor yaw responses in the blowfly Calliphora erythrocephala. Proc. R. Soc. Lond. B Biol. Sci. 219: 211-216, 1983.

HaUSEN, K. AND WeHRHAHN, C. Neural circuits mediating visual flight in flies. II. Separation of two control systems by microsurgical brain lesions. J. Neurosci. 10: 351-360, 1990.

Hausen, K., Wolburg-Buchholz, K., and Ribi, W. A. The synaptic organization of visual interneurons in the lobula complex of flies. Cell Tissue Res. 208: 371-387, 1980.

HENGSTENBERG, R. AND HENGSTENBERG, B. Intracellular staining of insect neurons with procion yellow. In: Neuroanatomical Techniques, edited by N. J. Strausfeld and T. A. Miller. Berlin: Springer-Verlag, 1980, p. $307-324$. 
IBBOTSON, M. R. Wide-field motion-sensitive neurons tuned to horizontal movement in the honeybee, Apis mellifera. J. Comp. Physiol. A 168 91-102, 1991 .

Kolb, H. AND Nelson, R. Amacrine cells of the cat retina. Vision Res. 21 1625-1633, 1981 .

MulleR, K. J. AND MCMAHAN, U. J. The shapes of sensory and motor neurones and the distribution of their synapses in ganglia of the leech: a study using intracellular injection of HRP. Proc. R. Soc. Lond. B Biol. Sci. 194: 481-499, 1976.

Poggio, T., Reichardt, W., and Hausen, K. A neuronal circuitry for relative movement discrimination by the visual system of the fly. Naturwissenschaften 68: 443-446, 1981.

Reichardt, W., EgelhaAf, M., AND Guo, A. Processing of figure and background motion in the visual system of the fly. Biol. Cybern. 61: 327-345, 1989.

REICHARDT, W. AND POGGIO, T. Figure-ground discrimination by relative movement in the visual system of the fly. Part I. Experimental results. Biol. Cybern. 35: 81-100, 1979.

Reichard, W., Poggio, T., and Hausen, K. Figure-ground discrimination by relative movement in the visual system of the fly. Part II. Towards the neural circuitry. Biol. Cybern. 46, Suppl.: 1-30, 1983.
SHePHERD, G. M. The olfactory bulb: a simple system in the mammalian brain. In: Handbook of Physiology. The Nervous System. Cellular Biology of Neurons. Bethesda, MD: Am. Physiol. Soc., 1977, sect. 1, vol. 1, p. 945-968.

Strausfeld, N. J. Atlas of an Insect Brain. Berlin: Springer-Verlag, 1976.

Strausfeld, N. J. AND Gilbert, C. Small-field neurons associated with oculomotor control in muscoid flies: cellular organization in the lobula plate. J. Comp. Neurol. 316: 56-71, 1992.

VAney, D. I., Collin, S. P., And Young, H. M. Dendritic relationship between cholinergic amacrine cells and direction-selective ganglion cells. In: Neurobiology of the Inner Retina, edited by R. Weiler and N. N. Osborne. Berlin: Springer-Verlag, 1989, p. 157-168.

Warzecha, A.-K., EgelhaAf, M., AND Borst, A. Neural circuit tuning fly visual interneurons to motion of small objects. I. Dissection of the circuit by pharmacological and photoinactivation techniques. J. Neurophysiol. 69: 329-339, 1993.

Watson, A. H. D. AND BURROws, M. The ultrastructure of identified locust motor neurones and their synaptic relationships. J. Comp. Neurol. 205: 383-397, 1982. 